\title{
Nutrizione parenterale intradialitica in pazienti con malnutrizione moderata-severa: studio prospettico osservazionale multicentrico
}

\author{
Concetto Sessa ${ }^{1}$, Walter Morale ${ }^{1}$, Antonino Reina ${ }^{2}$, Giorgio Battaglia ${ }^{2}$, Sandra La Rosa ${ }^{3}$, Daniela Puliatti ${ }^{4}$, \\ Giuseppe Seminara ${ }^{4}$, Luca Zanoli ${ }^{5}$ \\ ${ }^{1}$ U.O.C. Nefrologia e Dialisi, P.O. "Maggiore" di Modica, Ragusa - Italy \\ ${ }^{2}$ U.O.C. Nefrologia e Dialisi, P.O. S. Marta e S. Venera, Acireale (CT) - Italy \\ "U.O. Nefrologia e Dialisi, P.O. "Giovanni Paolo II" Sciacca, Agrigento - Italy \\ "U.O.C. Nefrologia, A.O. per l'Emergenza "Cannizzaro", Catania - Italy \\ ${ }^{5}$ Nefrologia, Dipartimento di Medicina Clinica e Sperimentale, Università di Catania, Catania - Italy
}

\begin{abstract}
Intradialytic parenteral nutrition in patients with moderate-severe malnutrition: a multicentre experience
Dialysis patients have a wide range of pathologies that contribute to their frailty. Maintaining a good nutritional status is useful to prevent and treat the so-called Protein-Energy Wasting (PEW), a complex clinical-laboratory condition in which a protein-energy depletion occurs. Adherence to a proper nutritional therapy in CKD requires considerable effort from both patients and health personnel (doctors and nurses). In order to slow down the effects of malnutrition and the disasters that complicate PEW, nephrologists can use supplementation products. In our observational, prospective, multicentre study, we administered an intradialytic parenteral nutrition of a three-compartment emulsion for intravenous infusion through an infusion pump connected to the venous line. After 12 weeks of treatment, subjects with severe malnutrition were reduced from $61.1 \%$ to $33.3 \%$, serum creatinine increased by $16 \%$ (from $6.00 \pm 1.48 \mathrm{mg} / \mathrm{dL}$ to $6.98 \pm 2.46 \mathrm{mg} / \mathrm{dL} ; \mathrm{P}<0.001$ ), total protein and albumin levels respectively by $13 \%$ (from $5.46 \pm 0.63 \mathrm{~g} / \mathrm{dL}$ to $6.19 \pm 0.66 \mathrm{~g} / \mathrm{dL}$; $\mathrm{P}<0.001$ ) and $19 \%$ (from $2.70 \pm 0.48 \mathrm{~g} / \mathrm{dL}$ to 3.20 $\pm 0.57 \mathrm{~g} / \mathrm{dL} ; \mathrm{P}<0.001$ ), body weight by $3 \%$ (from $55.7 \pm 13.2 \mathrm{~kg}$ to $57.6 \pm 13.0 \mathrm{~kg} ; \mathrm{P}<0.001$ ).
\end{abstract}

Keywords: Dialysis, Intradialytic parenteral nutrition, Malnutrition, Nutritional supplementation, Protein energy wasting

\section{Chiave di lettura \\ Ragionevoli certezze}

Tra i pazienti con insufficienza renale cronica (CKD) in trattamento emodialitico, la Protein Energy Wasting (PEW) può essere considerata una delle principali complicanze legate all'alimentazione. La sua patogenesi è multifattoriale e l'approccio terapeutico verte su una valutazione accurata e periodica. Quando l'assunzione di proteine e calorie è ridotta, il completamento nutrizionale per mezzo di formulazioni dedicate e somministrate durante la sessione di emodialisi

Received: August 30, 2021

Accepted: August 30, 2021

Published online: September 16, 2021

Indirizzo per la corrispondenza:

Concetto Sessa

U.O.C. Nefrologia

Ospedale "Maggiore"

Via Aldo Moro 1

97015 Modica (RG) - Italy

concettosessa@gmail.com può rappresentare un valido approccio alla prevenzione e al trattamento della PEW in pazienti selezionati.

\section{Aspetti controversi}

La PEW mostra un'elevata prevalenza tra i pazienti in trattamento emodialitico cronico ed è associata ad alti tassi di morbidità/mortalità oltre che all'incremento dei costi sanitari. La supplementazione con soluzioni nutrizionali al paziente, praticata direttamente durante la dialisi, può garantire benefici terapeutici aggiuntivi, sebbene non rappresenti, da sola, la soluzione al problema e sia da considerare un'integrazione all'uso di supplementi orali.

\section{Prospettive}

La correlazione tra miglioramento dello stato di malnutrizione, documentato oggettivamente mediante l'incremento della creatininemia, dell'albuminemia, della proteinemia totale e dell'emoglobina (in assenza di variazioni di Kt/V), e il miglioramento della qualità di vita del paziente, documentato 
attraverso il questionario Short Form 36-Item Health Survey (SF36), dopo IDPN (Intradialytic Parenteral Nutrition) suggerisce che questa può essere una valida opzione terapeutica per il nefrologo in presenza di malnutrizione. Sono, comunque, richiesti ulteriori studi che coinvolgano un campione più ampio e rappresentativo della popolazione emodializzata.

\section{Introduzione}

I pazienti sottoposti a trattamento sostitutivo della funzione renale presentano un rischio più alto di sviluppare malnutrizione sia rispetto alla popolazione generale che ai pazienti con stadio CKD I-IV (1). Recentemente, I'International Society of Renal Nutrition and Metabolism (ISRNM) ha introdotto la definizione "Deplezione Proteico-Energetica", in inglese Protein-Energy Wasting (PEW), per descrivere la progressiva riduzione del patrimonio proteico ed energetico dell'individuo (2). La prevalenza della PEW oscilla tra il $28 \%$ e il $48 \%$ per i pazienti con CKD stadio I-IV e varia tra il $50 \%$ e il $75 \%$ (3), con una prevalenza del $5-10 \%$ per la malnutrizione severa tra i pazienti con CKD stadio $V$ in trattamento emodialitico $(4,5)$. La patogenesi della PEW negli emodializzati è multifattoriale, riconducibile all'inadeguata assunzione di calorie e di proteine e alla perdita di nutrienti durante il trattamento dialitico in pazienti già "fragili" (3). Si tratta di un problema clinico spesso non riconosciuto e, talvolta, negato, che comporta un aumento della morbidità e un peggioramento della qualità di vita, un aumento delle ospedalizzazioni e un rallentamento dei processi riabilitativi e un aumento della mortalità e dei costi sanitari e sociali.

Lo spettro della PEW si fa più marcato nel paziente geriatrico a causa della presenza di diversi fattori concomitanti, quali le malattie croniche, la politerapia farmacologica (che influenza negativamente gli introiti e l'utilizzazione ottimale dei nutrienti), l'edentulia, il calo delle riserve fisiologiche per i processi di invecchiamento e la maggiore suscettibilità a complicanze acute. Inoltre, il paziente geriatrico è vittima del progressivo ridursi dell'autonomia funzionale, di problemi psicologici e di isolamento sociale ed economico, fattori che finiscono per esacerbare il problema della malnutrizione.

Indici dello scadimento dello stato nutrizionale del paziente sono da ricercare tra parametri biochimici (creatinina, proteine totali, albumina), antropometrici (peso, BMI) e bioimpedenziometrici (acqua corporea totale, indice di massa magra, indice di massa grassa).

Al fine di rallentare la PEW o di ridurne il rischio (2), il nefrologo può intervenire utilizzando prodotti di integrazione che, anche se non sono in grado di sostituire il pasto, permettono la supplementazione di nutrienti essenziali e un apporto calorico $(6,7)$. Questi integratori sono presenti in formulazioni per via orale e in emulsioni praticabili per via endovenosa durante il trattamento emodialitico.

Lo scopo del presente studio, condotto in pazienti con malnutrizione moderata-severa afferenti a 3 ambulatori di emodialisi, è stato la valutazione degli effetti della nutrizione parenterale intradialitica su indici di nutrizione facilmente reperibili in un ambulatorio di emodialisi e sulla sfera emozionale del paziente (indagata mediante questionario).

\section{Materiali e Metodi}

\section{Disegno dello studio}

Studio multicentrico, prospettico osservazionale e openlabel. Previa acquisizione del consenso informato, ciascun paziente sottoposto a emodialisi cronica da almeno sei mesi, è stato valutato al tempo zero (giorno dell'arruolamento) e dopo 12 settimane (36 trattamenti con l'emulsione parenterale intradialitica).

Prima dell'avvio dello studio, è stato predisposto un modulo per la raccolta e la segnalazione di eventi avversi distinti in gravi e non gravi, inserito nella cartella dialitica.

\section{Criteri di inclusione}

Sono stati arruolati pazienti adulti, di età superiore ai 18 anni di entrambi i sessi, con End Stage Kidney Disease (ESKD) in trattamento emodialitico cronico presso 3 centri di emodialisi nella provincia di Siracusa e a rischio di PEW, definito sulla base della scala di valutazione Mini Nutritional Assessment (MNA) $(8,9)$ La MNA consiste in un algoritmo progettato per un facile utilizzo da parte di medici di medicina generale, di infermieri e di operatori sanitari coinvolti nella valutazione nutrizionale dei pazienti. II test può essere eseguito in circa 10 minuti e comprende misurazioni semplici e un breve questionario. Prevede la valutazione antropometrica (peso, altezza e perdita di peso), la valutazione generale (stile di vita, terapia e mobilità), la valutazione dietetica (numero di pasti, assunzione di cibo e liquidi) e l'autovalutazione del paziente circa la propria percezione della salute e della nutrizione. In virtù dei risultati ottenuti, sono stati arruolati i soggetti a rischio di malnutrizione (punteggio 23,5-17) o in cattivo stato di nutrizione (punteggio $<17$, BMI $<19$ e circonferenza brachiale $\leq 20 \mathrm{~cm}$ per soggetti di sesso maschile e $<18 \mathrm{~cm}$ per soggetti di sesso femminile) (10). Sono stati, invece, esclusi dallo studio i pazienti che presentavano (a) ipersensibilità all'uovo, al pesce, alle arachidi, alla soia o a uno qualsiasi degli eccipienti presenti nel prodotto (acido citrico monoidrato, lecitina d'uovo, glicerolo, sodio oleato, all-rac- $\alpha$-tocoferolo), (b) disturbi congeniti del metabolismo degli aminoacidi, (c) iperlipidemia grave (trigliceridi $>500 \mathrm{mg} / \mathrm{dL}$ o LDL $>100 \mathrm{mg} /$ $\mathrm{dL})$, (d) iperglicemia non rispondente a dosi di insulina fino a 6 unità di insulina/ora, (e) grave insufficienza epatica (CHILDPUGH III-IV), (f) diatesi emorragica ingravescente, (g) embolia lipidica, (h) condizioni di collasso e shock, (i) infarto miocardico o ictus, (l) iperpotassiemia o iponatriemia, $(\mathrm{m})$ edema polmonare, $(\mathrm{n})$ insufficienza cardiaca scompensata e (o) infezione batterica in corso o una storia di infezione batterica negli ultimi 30 giorni. 


\section{Sacche per Nutrizione Parenterale Intradialitica}

È stata somministrata ai soggetti arruolati un'emulsione per infusione endovenosa tricompartimentale tramite pompa infusionale collegata alla linea venosa della dialisi. La sacca, pronta all'uso dopo miscelazione del contenuto dei singoli compartimenti, permette l'infusione, in $625 \mathrm{~mL}$ di volume di soluzione, di $740 \mathrm{kcal}$ distribuite nei tre compartimenti glicidico, lipidico e aminoacidico.

\section{Valutazioni}

Al basale e a 12, 24 e 36 trattamenti, ciascun soggetto è stato sottoposto a diverse valutazioni, che comprendevano il prelievo venoso per l'indagine biochimica e una valutazione dello stato nutrizionale.

Valutazione biochimica. I soggetti sono stati sottoposti a prelievo venoso per azoto ureico, Blood Urea Nitrogen (BUN) PRE/POST dialisi (per la stima del Kt/V con la formula di Daugirdas), glicemia capillare, creatininemia, esame emocromocitometrico e protidogramma (per valutazione dell'albuminemia e delle proteine totali).

Valutazione generale dello stato nutrizionale. In ciascun soggetto sono stati acquisiti, al basale e dopo 36 trattamenti, peso corporeo, body mass index (BMI), esame bioimpedenziometrico, scala di valutazione MNA, transferrinemia, proteina $C$ reattiva e questionario SF36 per la valutazione del benessere soggettivo (11).

\section{Bioimpedenziometria}

La bioimpedenziometria è stata eseguita con il Body Composition Monitor $\left(\mathrm{BCM}^{\circledR}\right.$, Fresenius Medical Care AG \& Co. KGaA, 61346 Bad Homburg v.d.H., Germany). Questo dispositivo si basa sulla bioimpedenza a spettroscopia (BIS), che misura l'impedenza generata da 50 correnti a frequenze fra $5 \mathrm{KHz}$ e $1 \mathrm{MHz}$, che percorrono il tratto compreso fra gli elettrodi posizionati sul piede e sulla mano del paziente. Con questa apparecchiatura, abbiamo stimato lo stato di idratazione e la composizione corporea, compresi la quota di acqua extra- e intracellulare, la quota di iperidratazione e gli indici di massa magra (LTI) e di massa grassa (FTI). II LTI, ottenuto dal rapporto tra massa magra (LTM) e altezza del soggetto (LTM/ altezzaㄹ), consente di approssimare la massa cellulare attiva e dà informazioni su possibili deficit nell'assunzione di proteine. II FTI è il complesso di grasso nell’organismo.

\section{Protocollo}

Ciascun soggetto arruolato nello studio dopo la valutazione nutrizionale MNA e l'anamnesi allergologica è stato sottoposto, durante il colloquio medico, a questionario SF36 sulla percezione della propria qualità di vita, alla registrazione del peso (e dei parametri vitali) in cartella e delle comorbidità secondo lo schema proposto dal Charlson Index (fatta eccezione per lo scompenso cardiaco e l'epatopatia grave, che controindicano la somministrazione di IDPN, e per la malattia renale, in quanto tutti i pazienti arruolati presentavano insufficienza renale allo stato terminale), al prelievo venoso e alla bioimpedenziometria. Inoltre, dopo aver identificato i pazienti a rischio di malnutrizione mediante MNA, si è provveduto a stratificare in maniera oggettiva l'entità della malnutrizione eseguendo la valutazione globale soggettiva (Subjective Global Assessment, SGA) sulla base del cambiamento del peso corporeo, dell'assunzione di alimenti, della comparsa di sintomi gastrointestinali e della diminuzione del grasso sottocutaneo e dell'atrofia muscolare (12). Ai soggetti arruolati (o a un tutore, qualora essi non si trovassero nelle condizioni psicofisiche di apporre la propria firma), veniva data lettura della nota informativa relativa agli allergeni contenuti nelle sacche, ai rischi e ai benefici derivanti dal trattamento e veniva richiesta la sottoscrizione del consenso informato.

Quindi, per ciascuna seduta, dopo la determinazione del peso di attacco, si procedeva a collegare il set di infusione alla linea venosa e (a) a impostare 4 ore di dialisi (al fine di garantire la somministrazione della quota di kcal nota), (b) ad aggiungere nel calo peso impostato nel monitor di dialisi il volume di nutrizione parenterale che si prevede di infondere (totale $625 \mathrm{~mL}$ ), (c) a incrementare la velocità di infusione gradualmente $(50 \mathrm{~mL} / \mathrm{h}$ per 15 minuti e $100 \mathrm{~mL} / \mathrm{h}$ per i successivi 15 minuti. A seguire impostare la pompa di infusione alla velocità di $150 \mathrm{~mL} / \mathrm{h}$ ), (d) a evitare la cosomministrazione di farmaci nel pozzetto venoso e (e) in presenza di segni di anafilassi, non riscontrata in alcun soggetto arruolato in questo studio, alla sospensione immediata del trattamento. La pressione arteriosa e i parametri vitali sono stati raccolti ogni ora (o, più frequentemente, a discrezione del personale presente in sala dialisi). Le caratteristiche della composizione della sacca e le quantità dei diversi nutrienti somministrate per seduta sono state riassunte nella Tabella I.

\section{Analisi Statistica}

I dati sono presentati come media \pm DS o percentuale. Le variabili considerate sono state analizzate attraverso l'analisi della varianza per dati ripetuti, mediante software NCSS 2007 \& PASS 11 (Gerry Hintze, Kaysville, Utah, USA).

\section{Risultati}

Questo studio è stato condotto tra giugno 2015 e luglio 2017 in pazienti con ESKD afferenti a 3 ambulatori di emodialisi della provincia di Siracusa. Tra gli 86 pazienti emodializzati presso i tre centri di emodialisi reclutati per lo studio (maschi $68 \%$, età $75 \pm 9$ anni), i pazienti con $M N A<23,5$ risultavano 
TABELLA I - Composizione Qualitativa E Quantitativa Della Sacca Per IDPN

\begin{tabular}{|c|c|}
\hline Glucosio monoidrato & $99,0 \mathrm{~g}$ \\
\hline equivalente a glucosio anidro & $90,0 \mathrm{~g}$ \\
\hline Sodio diidrogeno fosfato diidrato & $1,560 \mathrm{~g}$ \\
\hline Zinco acetato diidrato & 4,39 mg \\
\hline Trigliceridi a catena media & $12,5 \mathrm{~g}$ \\
\hline Olio di soia raffinato & $10,0 \mathrm{~g}$ \\
\hline Acidi Grassi omega -3 & $2,5 \mathrm{~g}$ \\
\hline Isoleucina & $2,06 \mathrm{~g}$ \\
\hline Leucina & $2,74 \mathrm{~g}$ \\
\hline Lisina cloridrato & $2,49 \mathrm{~g}$ \\
\hline equivalente a lisina & $1,99 \mathrm{~g}$ \\
\hline Metionina & $1,71 \mathrm{~g}$ \\
\hline Fenilalanina & $3,08 \mathrm{~g}$ \\
\hline Treonina & $1,59 \mathrm{~g}$ \\
\hline Triptofano & $0,50 \mathrm{~g}$ \\
\hline Valina & $2,26 \mathrm{~g}$ \\
\hline Arginina & $2,37 \mathrm{~g}$ \\
\hline Istidina cloridrato monoidrato & $1,48 \mathrm{~g}$ \\
\hline equivalente a istidina & $1,10 \mathrm{~g}$ \\
\hline Alanina & $4,25 \mathrm{~g}$ \\
\hline Acido aspartico & $1,32 \mathrm{~g}$ \\
\hline Acido glutammico & $3,07 \mathrm{~g}$ \\
\hline Glicina & $1,45 \mathrm{~g}$ \\
\hline Prolina & $2,98 \mathrm{~g}$ \\
\hline Serina & $2,63 \mathrm{~g}$ \\
\hline Sodio idrossido & $0,732 \mathrm{~g}$ \\
\hline Sodio cloruro & $0,237 \mathrm{~g}$ \\
\hline Sodio acetato triidrato & $0,157 \mathrm{~g}$ \\
\hline Potassio acetato & $2,306 \mathrm{~g}$ \\
\hline Magnesio acetato tetraidrato & $0,569 \mathrm{~g}$ \\
\hline Calcio cloruro diidrato & $0,390 \mathrm{~g}$ \\
\hline Contenuto di aminoacidi & $35,1 \mathrm{~g}$ \\
\hline Contenuto totale di azoto & $5 \mathrm{~g}$ \\
\hline Contenuto di carboidrati & $90 \mathrm{~g}$ \\
\hline Contenuto lipidico & $25 \mathrm{~g}$ \\
\hline Sodio & $33,5 \mathrm{mmol}$ \\
\hline Potassio & $23,5 \mathrm{mmol}$ \\
\hline Magnesio & $2,65 \mathrm{mmol}$ \\
\hline Calcio & $2,65 \mathrm{mmol}$ \\
\hline Zinco & $0,02 \mathrm{mmol}$ \\
\hline Cloruro & $30 \mathrm{mmol}$ \\
\hline Acetato & $30 \mathrm{mmol}$ \\
\hline Fosfato & $10 \mathrm{mmol}$ \\
\hline Contenuto energetico in lipidi & 995 (240) [kJ (kcal)] \\
\hline Contenuto energetico in carboidrati & $1.510(360)[\mathrm{kJ}(\mathrm{kcal})]$ \\
\hline Contenuto energetico in aminoacidi & 590 (140) [kJ (kcal)] \\
\hline Contenuto energetico non proteico & $2.505(600)[\mathrm{kJ}(\mathrm{kcal})]$ \\
\hline Contenuto energetico totale & $3.095(740)[\mathrm{kJ}(\mathrm{kcal})]$ \\
\hline Osmolalità & $2.115[\mathrm{mOsm} / \mathrm{kg}]$ \\
\hline Osmolarità teorica & $1.545[\mathrm{mOsm} / \mathrm{kg}]$ \\
\hline $\mathrm{pH}$ & $5,0-6,0$ \\
\hline
\end{tabular}

essere 34 ( 27 maschi, età $74 \pm 7$ anni). Tra questi, 2 pazienti non hanno prestato il consenso all'infusione delle soluzioni nutrizionali, 2 pazienti sono stati ospedalizzati e successivamente persi al follow-up prima di iniziare il trattamento IDPN e 5 sono stati rigettati dai medici, in quanto, pur possedendo i criteri di inclusione, presentavano un incremento ponderale inter-dialitico così elevato da rendere i tassi di ultrafiltrazione oraria impossibili, se addizionati alle soluzioni IDPN.

Dei 25 pazienti considerati eleggibili, 4 sono stati esclusi per il possibile rischio di reazione allergica (anamnesi personale di allergie ad arachidi e frutta secca), 2 per grave insufficienza epatica (CHILD-PUGH III) e 1 è stato escluso per ipercolesterolemia familiare (con $\mathrm{LDL}>290 \mathrm{mg} / \mathrm{dL}$ ), mentre 18 presentavano i requisiti e sono stati arruolati in questo studio (Fig. 1). Di questi pazienti, il 78\% era costituito da maschi, età media $75 \pm 12$ anni (range 54-89 anni), età media dialitica 3,5 \pm 1,9 anni. Tra i soggetti arruolati, il 39\% veniva sottoposto a emodialisi attraverso CVC e il 61\% mediante fistola arterovenosa; tutti dializzavano in modalità emodialisi standard con filtri di helixone. II 39\% presentava una malnutrizione moderata (SGA B) e il $61 \%$ una malnutrizione severa (SGA C), con una riduzione media del peso corporeo negli ultimi sei mesi del 6\%. II 56\% dei pazienti arruolati era stato ospedalizzato nei 3 mesi prima dell'inizio dell'IDPN e il $44 \%$ nei sei mesi precedenti.

Dopo 36 settimane di trattamento con nutrizione parenterale intradialitica, i soggetti con malnutrizione severa (SGA C) si sono ridotti dal $61 \%$ al $33 \%$. Parallelamente, la creatininemia aumentava del $16 \%$ (da $6,00 \pm 1,48 \mathrm{mg} / \mathrm{dL}$ a $6,98 \pm$ $2,46 \mathrm{mg} / \mathrm{dL} ; \mathrm{P}<0,001$ ), mentre le proteine totali e l'albumina presentavano un incremento medio rispettivamente del $13 \%$ (da 5,46 $\pm 0,63 \mathrm{~g} / \mathrm{dL}$ a $6,19 \pm 0,66 \mathrm{~g} / \mathrm{dL} ; \mathrm{P}<0,001$ ) e del $19 \%$ (da 2,70 $\pm 0,48 \mathrm{~g} / \mathrm{dL}$ a 3,20 $\pm 0,57 \mathrm{~g} / \mathrm{dL} ; \mathrm{P}<0,001$ ). Lo stesso si è verificato per il peso corporeo, che è aumentato del $3 \%$ (da $55,7 \pm 13,2 \mathrm{~kg}$ a $57,6 \pm 13,0 \mathrm{~kg} ; \mathrm{P}<0,001$ ), mentre trascurabile è stata la variazione del BMI. Sia LTI che FTI sono aumentati durante il follow-up rispettivamente del 30\% (da 9,5 $\pm 4,1$ a $12,3 \pm 5,0 ; \mathrm{P}<0,001$ ) e del $21 \%$ (da 8,4 $\pm 2,8$ a 10,2 $\pm 3,8 ; \mathrm{P}=$ $0,03)$, così come l'emoglobina (+16\%, da $9,2 \pm 2,4 \mathrm{~g} / \mathrm{dL}$ a 10,7 $\pm 2,8$ g/dL; $P<0,001$. Fig. 2, Fig. 3, Tab. II).

II Kt/V dei pazienti arruolati è rimasto costante nel corso del trattamento con IDPN $(P=0,2)$. Anche la glicemia, campionata mediante glucometro, non ha subito variazioni significative nel corso delle misurazioni durante il periodo di osservazione (Fig. 4).

Infine, abbiamo riscontrato un miglioramento del benessere soggettivo espresso dalla compilazione del questionario SF36, con un incremento del 51\% (Tab. III) del punteggio medio $(P<0,001)$.

Nessuno dei soggetti ha manifestato reazioni allergiche oppure ospedalizzazioni nelle 12 settimane di trattamento. Al termine delle 12 settimane, in alcuni pazienti si è continuata a praticare I'IDPN in accordo con le necessità cliniche. Tuttavia, non sono disponibili ulteriori risultati a lungo termine, perché questo non è richiesto dal protocollo dello studio. 


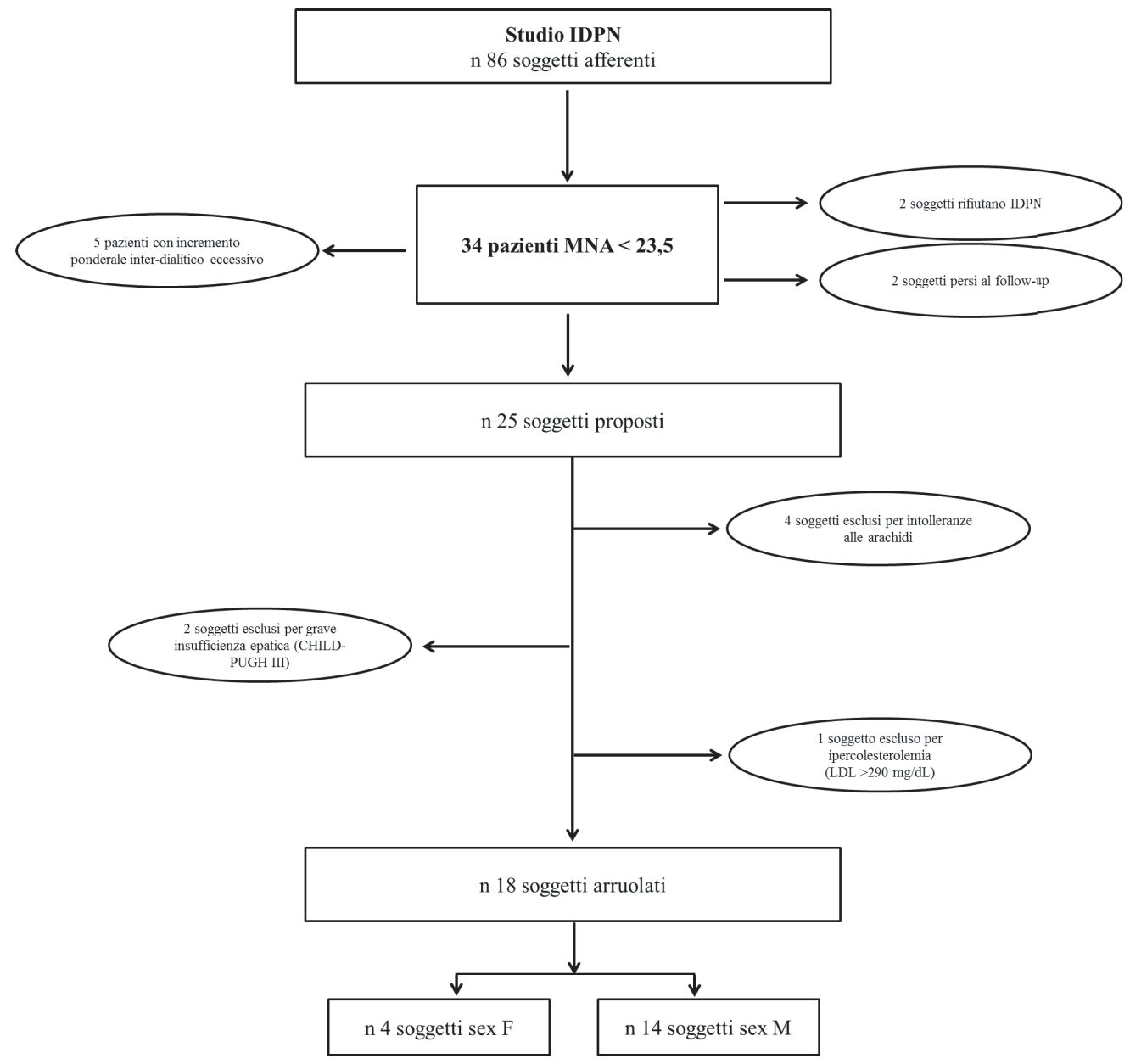

Fig. 1 - Flow-chart arruolamento soggetti allo studio.

\section{Discussione}

Il risultato principale del nostro studio riguarda la dimostrazione dell'utilità dell'IDPN nel migliorare l'apporto calorico-proteico dei pazienti malnutriti in dialisi, con riduzione della malnutrizione severa (SGA C). Tale miglioramento, documentato oggettivamente mediante l'incremento della creatininemia, dell'albuminemia e della proteinemia totale, del LTI, del FTI e dell'emoglobina, ha prodotto, inoltre, un consequenziale miglioramento soggettivo del paziente, documentato attraverso il questionario SF36.

II Kt/V rappresenta il metodo di riferimento per misurare la dose dialitica ed è definito come la clearance dell'urea (K) realizzata dal dializzatore moltiplicata per la durata del trattamento dialitico ( $\mathrm{t}$, in minuti) rapportata al volume di distribuzione dell'urea nell'organismo ( $\mathrm{V}$, in $\mathrm{mL})$, che è approssimativamente uguale all'acqua corporea totale, corretta per il volume perso durante l'ultrafiltrazione (13-15). II $\mathrm{Kt} / \mathrm{V}$ era stabile nel corso di tutto il periodo di studio, suggerendo che il miglioramento soggettivo della qualità di vita del paziente e il miglioramento dell'anemia e degli altri indicatori considerati erano indipendenti dall'efficienza dialitica.
Un basso apporto proteico è associato all'aumento della mortalità tra i pazienti in emodialisi (16). Le Linee Guida (17) raccomandano l'assunzione di $1,2 \mathrm{~g} / \mathrm{kg}$ di proteine al giorno (di cui almeno il $50 \%$ a elevato valore biologico) e da 30 a $35 \mathrm{kcal} / \mathrm{kg}$ al giorno. Il paziente emodializzato cronico è un paziente fragile, co-morbido, molto spesso esposto a fattori di rischio non modificabili e legati alla propria condizione di malato di rene ed emodializzato. L'isolamento e il tentativo di correggere la PEW riflettono il nostro approccio, che prevede una presa in carico del paziente di tipo olistico, finalizzata alla "fenotipizzazione" della terapia dialitica.

Fino al 2008, le alterazioni dello stato nutrizionale nel paziente nefropatico venivano definite con termini differenti, come malnutrizione, sarcopenia o cachessia, fornendo una descrizione solo parziale della problematica, senza definire in maniera compiuta i meccanismi che influenzano lo stato di salute e la prognosi di tali pazienti. L'uso di terminologie non uniformi e mal definite può portare sia a errori concettuali che a interpretazioni errate dei dati. Uniformare nomenclatura, definizioni e classificazioni, invece, significa realizzare un approccio più sistematico e razionale, sia in termini di ricerca che di gestione clinica (18). Per tali motivi, nel 2009, 


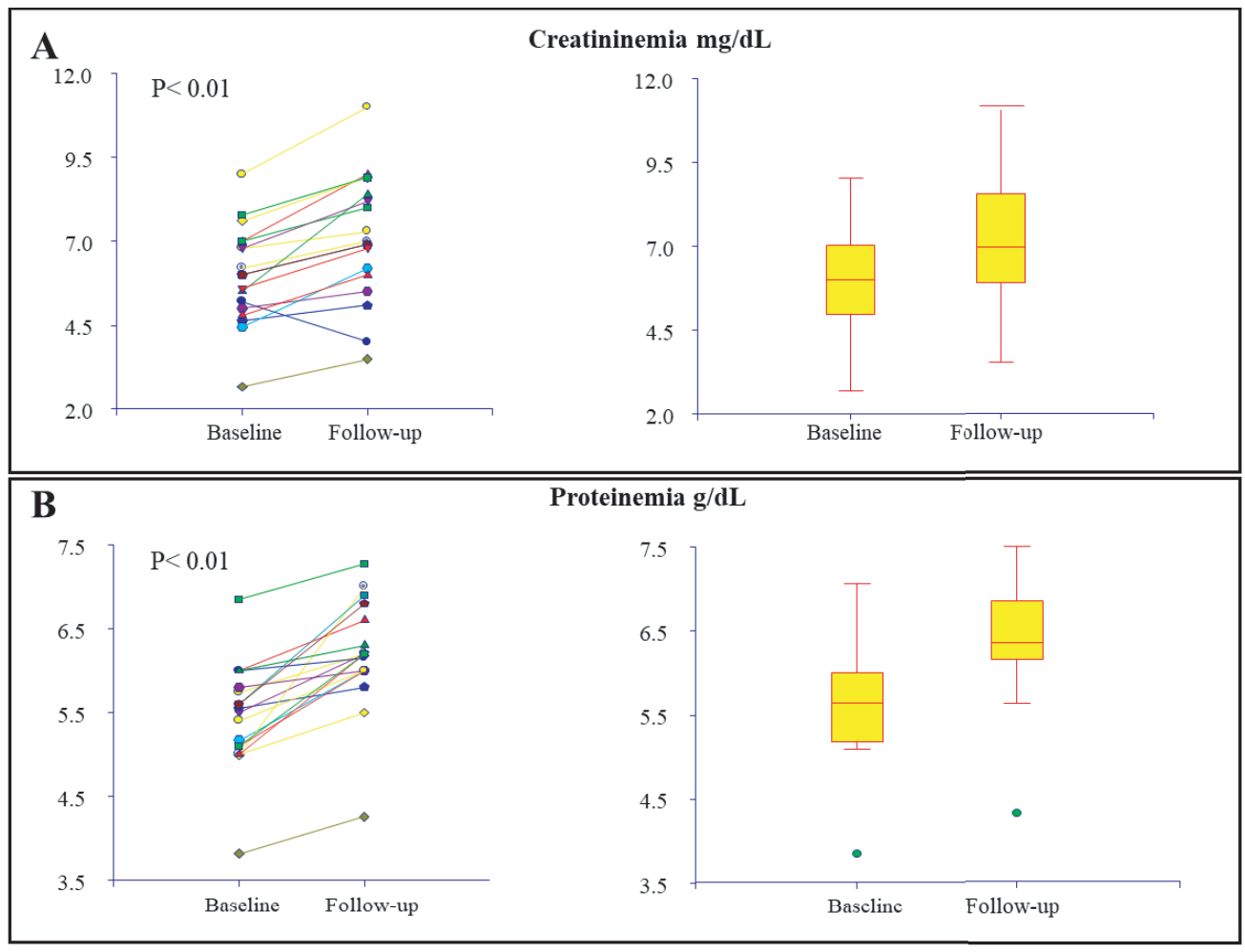

Fig. 2 - A: andamento della creatinina prima dell'IDPN e dopo; B: andamento delle proteine totali prima dell'IDPN e dopo; C: andamento dell'albumina prima dell'IDPN e dopo; D: andamento del peso prima dell'IDPN e dopo.

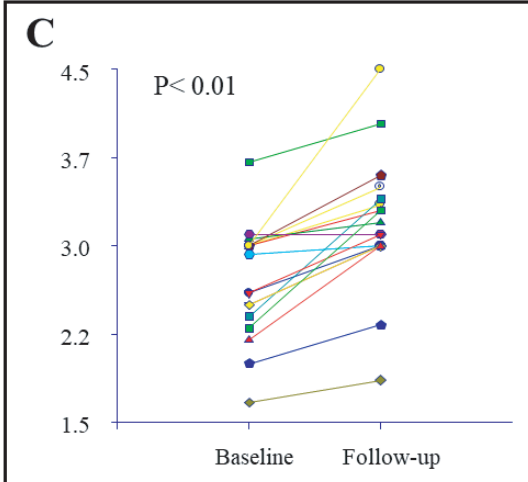

Albuminemia g/dL
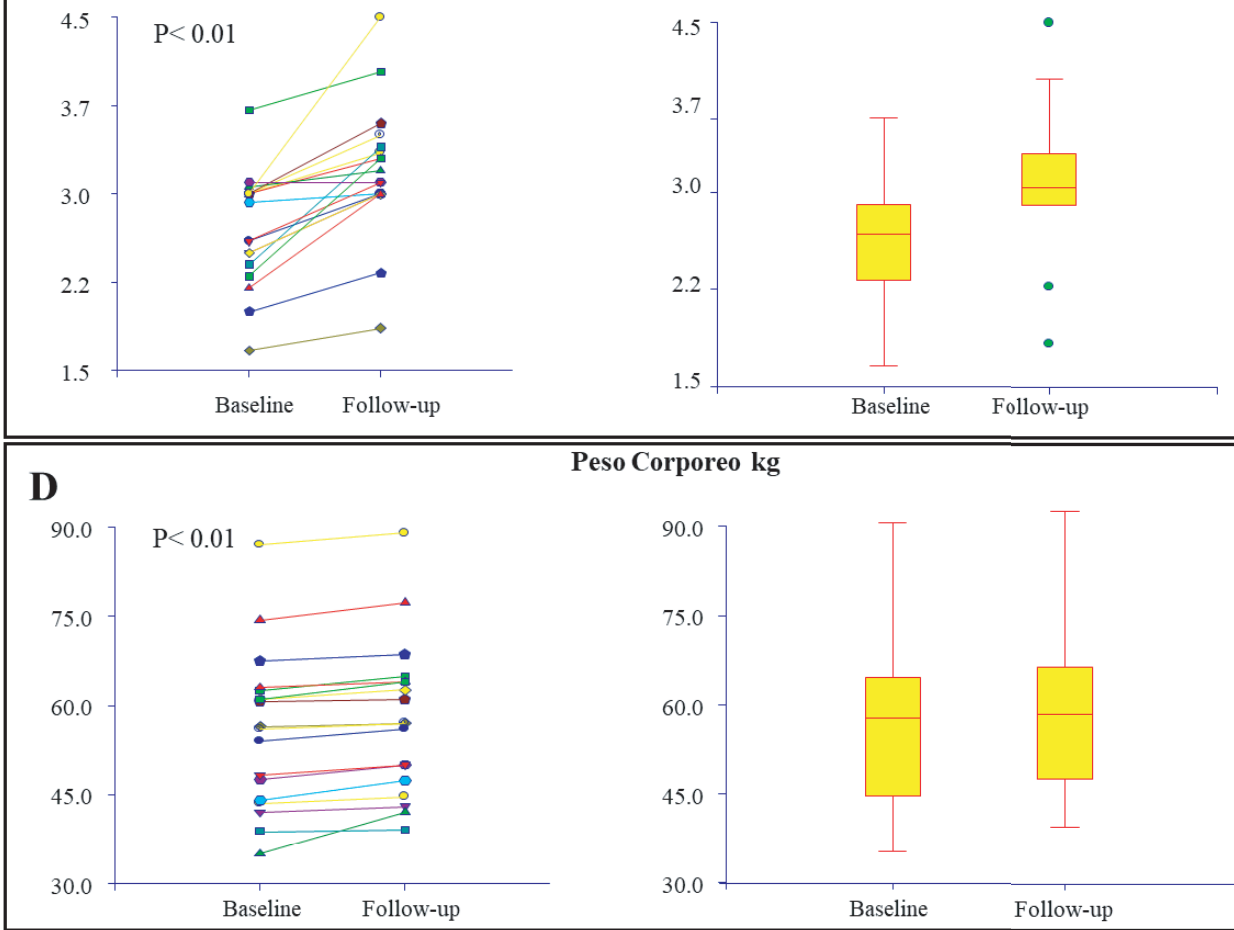

Peso Corporeo kg

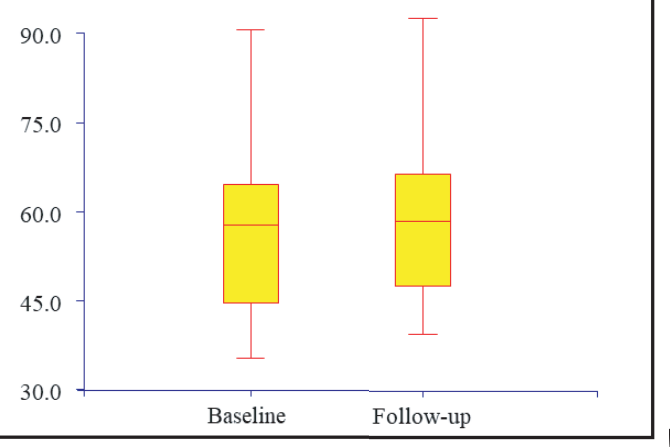

un gruppo di esperti appartenenti all'International Society of Renal Nutrition and Metabolism (ISRNM) ha individuato nella PEW I'unica espressione per definire la condizione clinica caratterizzata dalla presenza di alterazioni metabolico-nutrizionali e dalla progressiva compromissione del patrimonio proteico-energetico tipiche del paziente con malattia renale cronica (Chronic Kidney Disease, CKD) (19).

L'eziologia della PEW è multifattoriale e dipende da situazioni patologiche eterogenee (20). Tra queste, distinguiamo, per esempio, l'anoressia (21), che si presenta, di 


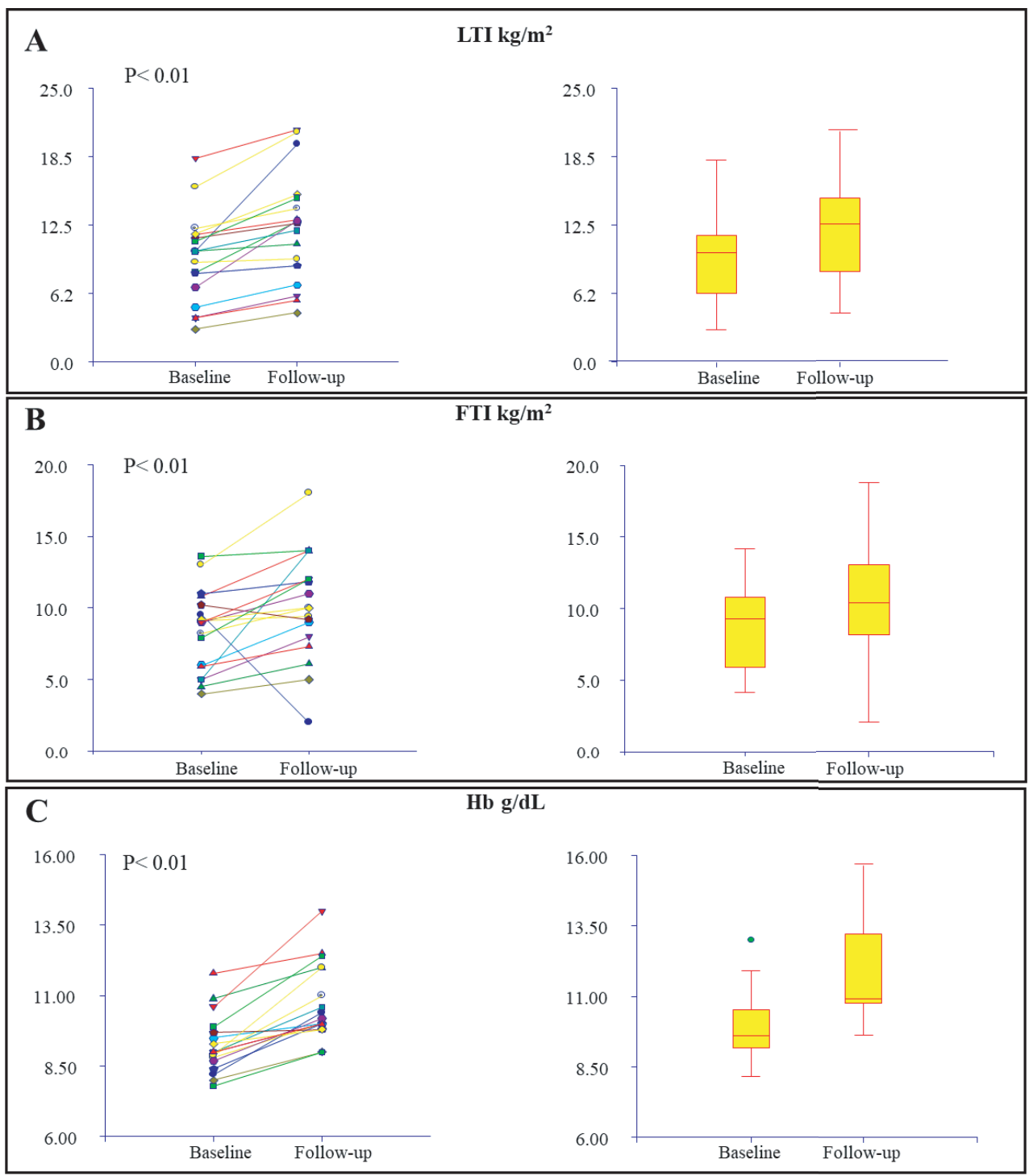

Fig. 3 - A: variazione dell'indice di massa magra (LTI) prima dell'IDPN e dopo; B: variazione dell'indice di massa grassa (FTI) prima dell'IDPN e dopo; C: variazione dell'emoglobina (Hb) prima dell'IDPN e dopo.

TABELLA II - Risultati dello studio IDPN effettuato dagli autori

\begin{tabular}{lccc}
\hline Parametri Valutati & $\begin{array}{c}\text { Basale } \\
\text { Media } \pm \text { DS }\end{array}$ & $\begin{array}{c}\text { Follow-up } \\
\text { Media } \pm \text { DS }\end{array}$ & P value \\
\hline Creatininemia (mg/dL) & $6,0 \pm 1,4$ & $6,9 \pm 2,4 \mathrm{mg} / \mathrm{dL}$ & $<0,001$ \\
Proteinemia (g/dL) & $5,46 \pm 0,63$ & $6,19 \pm 0,66 \mathrm{~g} / \mathrm{dL}$ & $<0,001$ \\
Albuminemia (g/dL) & $2,70 \pm 0,48$ & $3,20 \pm 0,57 \mathrm{~g} / \mathrm{dL}$ & $<0,001$ \\
Peso corporeo (kg) & $55,6 \pm 13,2$ & $57,6 \pm 12,9 \mathrm{~kg}$ & $<0,001$ \\
LTI (kg/m²) & $9,49 \pm 4,07$ & $12,33 \pm 5,03$ & $<0,001$ \\
FTI (kg/m $)$ & $8,38 \pm 2,84$ & $10,16 \pm 3,77$ & $<0,001$ \\
Hb (g/dL) & $9,2, \pm 2,4$ & $10,7 \pm 2,8 \mathrm{~g} / \mathrm{dL}$ & $<0,001$ \\
SF36 & $49 \pm 11$ & $74 \pm 13$ & $<0,001$ \\
\hline
\end{tabular}

LTI: indice di massa magra; FTI: indice di massa grassa; $\mathrm{Hb}$ : emoglobina; SF36: Short Form 36-Item Health Survey; IDPN: intradialytic parenteral nutrition. solito, nei pazienti emodializzati come frutto dello stato cronico dell'intossicazione uremica (che spesso comporta disgeusia) e della disregolazione degli ormoni gastroenterici (quali leptina e visfaptina) $(22,23)$. Allo scadimento dello stato nutrizionale, contribuisce anche l'infiammazione persistente, che, nel paziente con insufficienza renale cronica in trattamento emodialitico, è determinata da diverse cause, quali a) la diminuzione della clearance renale delle citochine proinfiammatorie, b) il sovraccarico di volume con conseguente aumento nell'assorbimento di endotossine (la congestione vascolare, dovuta al sovraccarico di liquidi, può provocare un'alterata permeabilità del tratto gastrointestinale, portando, così, all'accumulo di endotossine come lipopolisaccaridi e batteri. Questi processi possono, a loro volta, stimolare i monociti e l'aumento del rilascio di citochine proinfiammatorie), c) lo stress ossidativo e carbonilico (l'eccessiva produzione di radicali liberi o bassi 


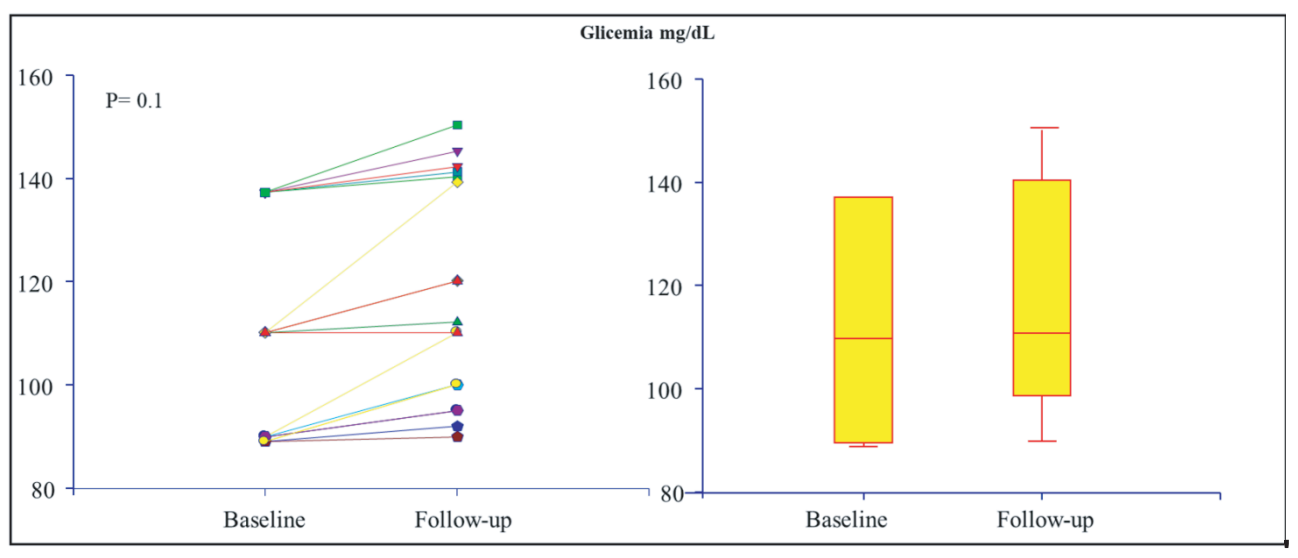

Fig. 4 - Variazione della glicemia prima e dopo IDPN.

TABELLA III - Variazione \% nel punteggio Short Form 36-Item Health Survey (SF36) per ciascun topic, dopo il trattamento con IDPN

\begin{tabular}{lc}
\hline Sezione SF36 & Variazione \% dopo IDPN \\
\hline Vitalità & $+15 \%$ \\
Funzionalità fisica & +14 \\
Dolore fisico & $+8 \%$ \\
Percezione della salute generale & $+11 \%$ \\
Ruolo fisico & $+9 \%$ \\
Ruolo emotivo & $+14 \%$ \\
Ruolo sociale & $+7 \%$ \\
Salute mentale & +11 \\
\hline
\end{tabular}

livelli di antiossidanti potrebbero rappresentare condizioni importanti per lo sviluppo di disfunzione endoteliale, infiammazione e aterogenesi) e d) la frequente presenza di condizioni di comorbidità nei pazienti renali, che aumenta lo stato ipercatabolico e, quindi, lo sviluppo dell'infiammazione (24). Inoltre, un potere pro-infiammatorio di per sé, legato all'esposizione a filtri e linee, alla scarsa qualità dell'acqua osmotizzata, alla back-filtration e alla presenza di materiale estraneo (come nel caso del politetrafluoroetilene dei cateteri venosi centrali) è fornito dal trattamento emodialitico stesso (25). L'acidosi metabolica viene riconosciuta da lungo tempo come causa esacerbante la malnutrizione, come conseguenza della spiccata tendenza dello stato acidosico alla degradazione spontanea delle proteine. Infine, non è da trascurare la perdita di nutrienti attraverso il trattamento emodialitico stesso. Durante ogni seduta dialitica con membrane a basso flusso, sono state, infatti, stimate una perdita di 4-9 g di aminoacidi liberi, se il paziente è a digiuno, e di 8-10 g, se il paziente sta mangiando, e una perdita di circa 2-3 g di peptidi. In media, la perdita di glucosio ammonta a 20-30 g per seduta dialitica, nel caso in cui il dialisato non contenga glucosio $(26,27)$. Durante i trattamenti emodialitici con membrane ad alto flusso, la perdita di aminoacidi liberi è di circa 8 g (20). In considerazione del fatto che uno dei meccanismi principali della PEW fra i pazienti in emodialisi è rappresentato dall'insufficiente assunzione di nutrienti, la supplementazione con IDPN è utile nella prevenzione e nel trattamento della PEW.

I vantaggi presentati dall'IDPN sono molteplici: (a) non necessita di un accesso vascolare dedicato e/o aggiuntivo, (b) evita il sovraccarico idrico e garantisce un elevato rapporto proteine-calorie, (c) può essere somministrata ad ogni sessione di dialisi e monitorata dal personale della dialisi e (d) i prodotti in commercio non necessitano di refrigerazione, hanno una validità di oltre due anni, analogamente agli altri dispositivi medici della dialisi, sono immediatamente somministrabili e non rendono necessarie logistiche specifiche presso i plessi ospedalieri.

Tra gli svantaggi, invece, riconosciamo l'insufficiente apporto calorico giornaliero, in quanto tale somministrazione può avvenire solo 3 volte alla settimana, e il costo aggiuntivo a quello già sostenuto con la seduta dialitica (prezzo di costo per 5 sacche, pari a 329,18 euro più iva).

\section{Problemi metodologici}

In questo studio, sono stati identificati diversi punti di forza e diversi limiti.

Tra i punti di forza, identifichiamo il disegno dello studio (multicentrico, prospettico osservazionale), che differisce dalla maggior parte di quelli retrospettivi riportati in letteratura (28-30); i parametri di malnutrizione utilizzati sono validati dalla letteratura (31-35); la qualità di vita è stata valutata con un questionario validato dalla letteratura (35).

Tra i limiti dello studio, consideriamo il numero esiguo di soggetti arruolati, che non ha reso possibile eseguire analisi dei diversi sottogruppi in base a trattamento, età e comorbidità ed eseguire un'analisi della sopravvivenza; non è stato previsto un gruppo di controllo, in quanto, considerando la condizione dei soggetti arruolati, non è stato ritenuto etico. 


\section{Conclusioni}

Secondo la nostra esperienza, anche se limitata nei numeri e nella durata, la nutrizione parenterale intradialitica sembra giocare un ruolo rilevante, sia in termini di miglioramento oggettivo del paziente documentato mediante l'incremento della creatininemia, del peso, dell'albuminemia, della proteinemia totale e dell'emoglobina che in termini di percezione di un miglioramento soggettivo documentato attraverso il questionario SF36. Tale tipologia di trattamento ben si concilia con la necessità di fenotipizzare la terapia dialitica alle esigenze metaboliche del paziente.

La tempestività di intervento è necessaria per assicurare una risposta immediata già all'esordio dello stato di malnutrizione, impedendo, così, il deterioramento dello stato di salute. Tuttavia I'IDPN non è ipotizzabile per il trattamento dei pazienti con cachessia che necessitano di un supporto totale o giornaliero e rimane confinata a quelli con malnutrizione moderata su cui stabilire un supporto nutrizionale "compensativo" e non sostitutivo.

\section{Disclosures}

Conflict of interest: The authors declare no conflict of interest. Financial support: This research received no specific grant from any funding agency in the public, commercial, or not-for-profit sectors.

\section{Bibliografia}

1. Druml W, Kierdorf HP. Parenteral nutrition in patients with renal failure. Guidelines on Parenteral Nutrition, Chapter 17. Ger Med Sc. 2009 Nov 18;7:Doc11. CrossRef PubMed

2. Fouque D, Kalantar-Zadeh K, Kopple J, et al. A proposed nomenclature and diagnostic criteria for protein-energy wasting in acute and chronic kidney disease. Kidney Int. 2008;73(4): 391-398. CrossRef PubMed

3. Locatelli F, Fouque D, Heimburger $O$, et al. Nutritional status in dialysis patients: a European consensus.Nephrol Dial Transplant. 2002 Apr e 11917047. 17(4):563-72. CrossRef

4. Sabatino A, Regolisti G, Antonucci E et al. Intradialytic parenteral nutrition in end-stage renal disease: practical aspects, indications and limits. J Nephrol. 2014 Aug e 24557877. 27(4): 377-83. CrossRef

5. Cianciaruso B, Brunori G, Kopple JD, et al. Cross-sectional comparison of malnutrition in continuous ambulatory peritoneal dialysis and hemodialysis patients. Am J Kidney Dis. 1995; 26(3):475-486. CrossRef PubMed

6. Obi Y, Qader H, Kovesdy CP, Kalantar-Zadeh K. Latest consensus and update on protein-energy wasting in chronic kidney disease. Curr Opin Clin Nutr Metab Care. 2015;18(3):254-262. CrossRef PubMed

7. Koppe L, Fouque D, Kalantar-Zadeh K. Kidney cachexia or proteinenergy wasting in chronic kidney disease: facts and numbers. J Cachexia Sarcopenia Muscle. 2019;10(3):479-484. CrossRef

8. Vellas B, Guigoz Y, Garry PJ, et al. The Mini Nutritional Assessment (MNA) and its use in grading the nutritional state of elderly patients. Nutrition. 1999;15(2):116-122. CrossRef PubMed

9. Cereda E. Mini nutritional assessment. Curr Opin Clin Nutr Metab Care. 2012;15(1):29-41. CrossRef PubMed

10. Gazzetta Ufficiale Della Regione Siciliana - Parte I n. 37; 9-82013.
11. Zouari L, Omri S, Turki S, et al. Quality of life in chronic hemodialysis patients: about 71 cases. Tunis Med. 2016 e 94(1): 40-45. PubMed

12. Detsky AS, McLaughlin JR Jr, Baker JP, et al. What is subjective global assessment of nutritional status? JPEN J Parenter Enteral Nutr. 1987;11(1):8-13. CrossRef PubMed

13. Daugirdas JT. Rapid methods of estimating Kt/V: three formulas compared. ASAIO Trans. 1990;36(3):M362-M364. PubMed

14. Farrington $K$, Davenport A. Would prescribing target Kt dose adjusted for body surface area improve hemodialysis outcomes? Kidney Int. 2016;90(6):1160-1162. CrossRef PubMed

15. Thomas A, Silver SA, Perl J, et al. The Frequency of Routine Blood Sampling and Patient Outcomes Among Maintenance Hemodialysis Recipients. Am J Kidney Dis. 2020;75(4):471-479. CrossRef PubMed

16. Huang $\mathrm{CX}$, Tighiouart $\mathrm{H}$, Beddhu $\mathrm{S}$, et al. Both low muscle mass and low fat are associated with higher all-cause mortality in hemodialysis patients. Kidney Int. 2010 Apr, 20072111, 77(7):624-9. CrossRef

17. Tattersall J, Martin-Malo A, Pedrini Let al. EBPG guideline on dialysis strategies. Nephrol Dial Transplant. 2007 May e 17507427., 22 Suppl 2:ii5-21. CrossRef PubMed

18. Stenvinkel P, Heimburger O, Lindholm B. Wasting, but not malnutrition, predicts cardiovascular mortality in end-stage renal disease. Nephrol Dial Transplant 2004 e 2181-2183. CrossRef

19. Bozzoli L, Sabatino A, Regolisti G et al. Deplezione proteicoenergetica e supplementazione nutrizionale nei pazienti in emodialisi cronica. G Ital Nefrol 2015 e 24, 32(5). PubMed

20. Bossola M, Tazza L, Vulpio C, et al. La malnutrizione nel paziente in emodialisi cronica: prevalenza, patogenesi, terapia. G Ital Nefrol. 2009;26(2):201-214. PubMed

21. Bossola M, Tazza L, Giungi $\mathrm{S}$ et al. Anorexia in hemodialysis patients: an update. G. Kidney Int. 2006 e 70(3):417. CrossRef

22. Cheung W, Yu PX, Little BM et al. Role of leptin and melanocortin signaling in uremia-associated cachexia. J Clin Invest. 2005 e 115(6):1659. CrossRef PubMed

23. Carrero JJ, Witasp A, Stenvinkel P, et al. Visfatin is increased in chronic kidney disease patients with poor appetite and correlates negatively with fasting serum amino acids and triglyceride levels. Nephrol Dial Transplant. 2010;25(3):901906. CrossRef PubMed

24. Shi $K$, Wang F, Jiang $H$, et al. Gut bacterial translocation may aggravate microinflammation in hemodialysis patients. Dig Dis Sci. 2014 Sep e 15., 59(9):2109-17. Epub 2014 May. CrossRef

25. Dukkipati R, Molnar MZ, Park J, et al. Association of vascular access type with inflammatory marker levels in maintenance hemodialysis patients. Semin Dial. 2014 Jul-Aug e 24118625 , 27(4):415-23. CrossRef

26. Conte F, Righetti M, Limido A. On behalf of the Lombardy patients. Submitted abstract to ERA-EDTA Congress, Amsterdam 2014.

27. Wolfson M. Use of intradialytic parenteral nutrition in hemodialysis patients. Am J Kidney 1994, e 23(6):856. CrossRef

28. Foulks CJ. An evidence-based evaluation of intradialytic parenteral nutrition. Am J Kidney 1999 e 33(1):186. CrossRef

29. Capelli JP, Kushner H, Camiscioli TC et al. Effect of intradialytic parenteral nutrition on mortality rates in end-stage renal disease care. Am J Kidney Dis. 1994 e 23(6):808. CrossRef

30. Abdulan IM, Onofriescu M, Stefaniu R, et al. The predictive value of malnutrition for functional and cognitive status in elderly hemodialysis patients. Int Urol Nephrol. 2019;51(1):155-162. CrossRef PubMed

31. Kayardi M, Icagasioglu S, Yilmaz A, et al. Serum leptin levels and malnutrition in patients with chronic renal failure. Saudi Med J. 2006 e 27(4):477-481. PubMed 
32. Muñoz-Pérez E, Espinosa-Cuevas MLÁ, Miranda-Alatriste PV, et al. Combined assessment of nutritional status in patients with peritoneal dialysis using bioelectrical impedance vectors and malnutrition inflammation score. Nutr Hosp. 2017 e 34(5):1125-1132. CrossRef

33. Wang WL, Liang S, Zhu FL, Liu JQ, Chen XM, Cai GY. Association of the malnutrition-inflammation score with anthropometry and body composition measurements in patients with chronic kidney disease. Ann Palliat Med. 2019;8(5):596-603. CrossRef PubMed
34. Teixeira Nunes F, de Campos G, Xavier de Paula SM, et al. Dialysis adequacy and nutritional status of hemodialysis patients. Hemodial Int. 2008;12(1):45-51. CrossRef PubMed

35. Liem YS, Bosch JL, Arends LR et al. Quality of life assessed with the Medical Outcomes Study Short Form 36-Item Health Survey of patients on renal replacement therapy: a systematic review and meta-analysis. Value Health. 2007 e 10(5). CrossRef PubMed 